\title{
Mechanistic Insights into Arterial Repair with Mesenchymal Stromal Cells
}

\author{
Editorial to: "Stem Cell Therapy for Arterial Restenosis: Potential Parameters \\ Contributing to the Success of Bone Marrow-Derived Mesenchymal Stromal Cells" by A. \\ Forte et al.
}

Peter J. Psaltis

Published online: 13 December 2011

(C) Springer Science+Business Media, LLC 2011

Despite the widespread use of drug-eluting stent technology, arterial restenosis remains an important complication following percutaneous revascularization procedures [1]. Viewed simply, it can be considered as a disproportionate reparative reaction to iatrogenic vascular injury, that is brought about by a complex process, underpinned by different molecular pathways and contributions from diverse cell types recruited to the vessel wall or resident locally within its mural layers [2]. Central pathophysiological factors comprise the dysregulated deposition and metabolism of extracellular matrix components, along with alterations in cellular proliferation, migration and transdifferentiation. These ultimately lead to excessive neointima formation and re-narrowing of the arterial lumen. Although its frequency has declined in recent years, the residual risk of restenosis after percutaneous and surgical revascularization has prompted ongoing evaluation of experimental strategies to prevent or treat its progression, including the administration of different progenitor cell types to facilitate normal arterial healing and avert aberrant, deleterious repair [3-7].

In general terms, the influence of progenitor cells on vascular health cannot be broadly summarized as adaptive or maladaptive, as it is dependent on both specific cell type and disease context. This is underscored by contrasting outcomes following the delivery of "endothelial progenitor cells" (EPCs) or smooth muscle progenitor cells in different small animal models of spontaneous atherosclerosis, vascular graft arteriopathy or vascular injury (wire or balloon) [8]. While there is cautionary evidence to suggest that systemic

\section{P. J. Psaltis $(\bowtie)$}

Division of Cardiovascular Diseases, Mayo Clinic,

200 First St SW,

Rochester, MN 55905, USA

e-mail: psaltis.peter@mayo.edu infusion of EPCs may accentuate atherosclerotic burden in ApoE-deficient mice and prime atheroma toward an inflammation-rich state [9], these cells have also been used to beneficial effect to modulate vascular remodeling after intimal disruption [10]. After arterial angioplasty and stenting, it is desirable to accelerate intimal re-endothelialization in order to limit inflammation and extracellular matrix deposition within the vessel wall and in turn avoid the development of neointimal hyperplasia and in-stent restenosis. Therefore infusions of pro-angiogenic or endothelial progenitor cells from different tissue sources (e.g. bone marrow $[\mathrm{BM}]$, blood, adipose) have been used in preclinical studies to promote rapid luminal endothelial recovery after vascular insult $[5,11,12]$. Others have also attempted to achieve this by designing vascular stents and grafts that readily attract EPC coverage through magnetic forces $[13,14]$ or by special coating with capture antibodies [15].

Beyond interest in the application of different EPC subsets, there has also been increasing recognition that mesenchymal stromal cells (MSCs) from BM or other tissue sources may be well-suited to confer reparative benefits in the setting of vascular wall injury [2]. Owing to their ease of isolation and ex vivo expansion, potential for allogeneic, "off-the-shelf" use and range of pro-angiogenic and myogenic properties, these cells have been extensively investigated for their utility in cardiovascular therapeutics [16], with favorable results after their delivery in ischemic $[17,18]$ and nonischemic myocardial disease [19]. In the setting of arterial repair and attenuation of restenosis after vascular insult, there has been some conjecture as to the beneficial potential of MSCs. Theoretical concern has been raised that these cells may have a pro-fibrotic effect on the vessel wall and that their ability to promote angiogenesis (e.g. through secretion of vascular endothelial growth factor 
[VEGF] and other cytokines) could contribute to deleterious expansion of adventitial vasa vasorum [2]. In one study that investigated intravenous delivery of MSCs seven days after femoral artery wire injury, injected cells were found to adhere readily to sites of endothelial disruption, ultimately contributing to the cellular composition of neointimal hyperplasia, with some indication for their smooth muscle cell transformation [4]. However for the most part, the weight of preclinical evidence in vascular injury models has supported the capacity of MSCs to facilitate early endothelial repair, modulate local inflammatory responses and reduce late smooth muscle cell proliferation and neointimal thickening [2].

To this end, Forte et al. have previously reported that systemic infusion of allogeneic BM MSCs resulted in attenuation of inward remodeling and luminal narrowing thirty days into a rodent model of carotid artery crush and arteriotomy [3]. This surgical model has the important distinction from wire or balloon injury in that it creates a greater degree of arterial wall trauma by disrupting both the internal and external elastic lamina. In this initial study, DiI-labeled MSCs were found to home to the injured arterial wall, progressively accumulating within the adventitia, perivascular tissue and to a lesser extent the media and intima, over the first week after delivery. This was accompanied by early modulation of different cytokines within the injured vessel, but not the contralateral, unaffected artery to which cells did not track. In particular, there was (1) an increase in the local expression of the immunosuppressive factor TGF- $\beta$, which may have anti-proliferative effects on smooth muscle cells and influence the differentiation of adventitial myofibroblasts, along with (2) reductions of the pro-inflammatory cytokines IL-1 $\beta$ and MCP-1, which may limit macrophage invasion in the healing arterial wall.

In the current issue of Cardiovascular Drugs and Therapy, the same authors further show that early after their delivery, MSCs induce pleiotropic effects on other inflammatory and angiogenic mediators that are also implicated in vascular healing and response to injury [20]. They describe an initial spike at day 3 in mRNA expression of VEGF in carotid arteries from cell-treated rats compared to those that received control medium, and a reduction in mRNA levels of Toll-like receptors (TLR) 2 and 4 that persisted until at least day 7. Furthermore serum levels of VEGF were also increased after cell transfer, while there were diminished titers of a range of soluble inflammation-related molecules (CXCL1, ICAM-1, L-Selectin, LIX/CXCL5, CCL5/ RANTES), as well as TIMP-1, which has complex actions on extracellular matrix remodeling, cell growth and death. Interestingly, MSC infusion was not found to impact on immunohistochemical measures of local oxidative stress or apoptosis in the injured vessels, but it was accompanied by a substantial increase in the presence of proliferating cells throughout the vessel wall, as determined by incorporation of BRDU. Almost universally, MSC-induced changes were more prominent at day 3 than day 7 , while later time-points were not assessed to determine the durability of cell effect. Forte et al. did not directly investigate how these alterations in different soluble mediators impacted on specific cellular and extracellular targets within the arterial wall, nor did they determine whether the vascular homing of MSCs constituted actual engraftment and differentiation into endothelial or smooth muscle cell subtypes. However, they surmise that MSCs are likely to exert immunomodulatory effects on different innate and adoptive inflammatory cell subtypes that are activated after arterial injury, a finding that is consistent with recent results from another study [21].

The paracrine and trophic properties of MSCs are well appreciated and have been given increasingly more mechanistic weight in terms of mediating tissue repair than the capacity of MSCs to undergo adoptive transdifferentiation [16]. In the case of postnatal arteries, recent discoveries have identified the presence of diverse progenitor cell populations resident throughout the different mural layers [8]. Local progenitor cells may provide an important early target for the paracrine influence of exogenous MSC transfer, in keeping with Forte et al.'s observation that cell delivery triggered an increase in proliferating cells within the vessel wall. Pro-mitogenic stimulation of intimal EPCs could theoretically help to initiate arterial endothelialization and in turn contribute to the ability of MSCs to mitigate restenosis, as reported in their previous study [3].

Overall, the major implication of Forte et al.'s studies is that MSC therapy may trigger important cues to reset the cascade of inflammatory signals that are activated in the initial stages of vascular healing and to prime other cell populations that participate in local arterial repair. This work complements the growing preclinical experience acquired with EPC transfer in animal models of vascular injury and broadens the therapeutic scope of MSCs, which have considerable clinical appeal because of their possible allogeneic application. It also provides a framework to develop strategies to optimize the vascular reparative properties of MSCs. These may include combining MSC delivery with other cell types [4], or augmenting the paracrine capacity of MSCs through techniques such as immunoselective enrichment [22], hypoxic or pharmacological preconditioning or genetic transfection. The latter approach recently has been used to enhance the ability of EPCs to inhibit neointima formation $[23,24]$.

Although recent data indicate that intravenous injection of MSCs may be safely tolerated [18], it is likely that their effectiveness could be further improved by directing cells more specifically to the actual site of arterial injury or revascularization. This could be achieved by direct infusion into the lumen of the target vessel, or by using novel approaches like magnetic tagging [25], ultrasound- 
generated acoustic radiation forces [26] or antibody capture to attract cells to the surface of the revascularized conduit (stent or graft). New catheter injection systems are also in development that will enable cells to be injected transluminally into the vascular adventitia and periadventitial space. Given the promising results from small animal experiments, it is highly probable that the investigation of MSC therapy will quickly progress to large animal and human studies of arterial injury, revascularization and restenosis in the near future.

Funding Sources Dr Psaltis has no conflicts of interest and is the recipient of a Post-doctoral Overseas Biomedical Fellowship from the National Health and Medical Research Council of Australia.

\section{References}

1. Garg S, Serruys PW. Coronary stents: current status. J Am Coll Cardiol. 2010;56:S1-42.

2. Forte A, Galderisi U, Cipollaro M, Cascino A. Mesenchymal stem cells: a good candidate for restenosis therapy? Current Vasc Pharmacol. 2009;7:381-93.

3. Forte A, Finicelli M, Mattia M, et al. Mesenchymal stem cells effectively reduce surgically induced stenosis in rat carotids. J Cell Physiol. 2008;217:789-99.

4. Wang $\mathrm{CH}$, Cherng WJ, Yang NI, et al. Late-outgrowth endothelial cells attenuate intimal hyperplasia contributed by mesenchymal stem cells after vascular injury. Arterioscler Thromb Vasc Biol. 2008;28:54-60.

5. Froehlich H, Gulati R, Boilson B, et al. Carotid repair using autologous adipose-derived endothelial cells. Stroke. 2009;40:1886-91.

6. Wang SH, Lin SJ, Chen YH, et al. Late outgrowth endothelial cells derived from Wharton jelly in human umbilical cord reduce neointimal formation after vascular injury: involvement of pigment epithelium-derived factor. Arterioscler Thromb Vasc Biol. 2009; 29:816-22.

7. Takahashi M, Suzuki E, Oba S, et al. Adipose tissue-derived stem cells inhibit neointimal formation in a paracrine fashion in rat femoral artery. Am J Physiol Heart Circ Physiol. 2010;298: H415-23.

8. Psaltis PJ, Harbuzariu A, Delacroix S, Holroyd EW, Simari RD. Resident vascular progenitor cells - diverse origins, phenotype, and function. J Cardiovasc Transl Res. 2011;4:161-76.

9. George J, Afek A, Abashidze A, et al. Transfer of endothelial progenitor and bone marrow cells influences atherosclerotic plaque size and composition in apolipoprotein E knockout mice. Arterioscler Thromb Vasc Biol. 2005;25:2636-41.

10. Padfield GJ, Newby DE, Mills NL. Understanding the role of endothelial progenitor cells in percutaneous coronary intervention. J Am Coll Cardiol. 2010;55:1553-65.

11. Werner N, Junk S, Laufs $U$, et al. Intravenous transfusion of endothelial progenitor cells reduces neointima formation after vascular injury. Circ Res. 2003;93:e17-24.
12. Gulati R, Jevremovic D, Peterson TE, et al. Autologous culturemodified mononuclear cells confer vascular protection after arterial injury. Circulation. 2003;108:1520-6.

13. Pislaru SV, Harbuzariu A, Agarwal G, et al. Magnetic forces enable rapid endothelialization of synthetic vascular grafts. Circulation. 2006;114:I314-8.

14. Pislaru SV, Harbuzariu A, Gulati R, et al. Magnetically targeted endothelial cell localization in stented vessels. J Am Coll Cardiol. 2006;48:1839-45.

15. Aoki J, Serruys PW, van Beusekom H, et al. Endothelial progenitor cell capture by stents coated with antibody against CD34: the HEALING-FIM (Healthy Endothelial Accelerated Lining Inhibits Neointimal Growth-First In Man) Registry. J Am Coll Cardiol. 2005;45:1574-9.

16. Psaltis PJ, Zannettino AC, Worthley SG, Gronthos S. Concise review: mesenchymal stromal cells: potential for cardiovascular repair. Stem Cells. 2008;26:2201-10.

17. Quevedo HC, Hatzistergos KE, Oskouei BN, et al. Allogeneic mesenchymal stem cells restore cardiac function in chronic ischemic cardiomyopathy via trilineage differentiating capacity. Proc Natl Acad Sci USA. 2009;106:14022-7.

18. Hare JM, Traverse JH, Henry TD, et al. A randomized, doubleblind, placebo-controlled, dose-escalation study of intravenous adult human mesenchymal stem cells (prochymal) after acute myocardial infarction. J Am Coll Cardiol. 2009;54:2277-86.

19. Psaltis PJ, Carbone A, Nelson AJ, et al. Reparative effects of allogeneic mesenchymal precursor cells delivered transendocardially in experimental nonischemic cardiomyopathy. JACC Cardiovasc Interv. 2010;3:974-83.

20. Forte A, Rinaldi B, Sodano L, et al. Stem cell therapy for arterial restenosis: potential parameters contributing to the success of bone marrow-derived mesenchymal stromal cells. Cardiovasc Drugs Ther 2012;26:this issue.

21. Shoji M, Oskowitz A, Malone CD, Prockop DJ, Pochampally R. Human mesenchymal stromal cells (MSCs) reduce neointimal hyperplasia in a mouse model of flow-restriction by transient suppression of anti-inflammatory cytokines. J Atheroscler Thromb. 2011;18:464-74.

22. Psaltis PJ, Paton S, See F, et al. Enrichment for STRO-1 expression enhances the cardiovascular paracrine activity of human bone marrow-derived mesenchymal cell populations. J Cell Physiol. 2010;223:530-40.

23. Li D, Yan D, Liu W, et al. Foxc2 overexpression enhances benefit of endothelial progenitor cells for inhibiting neointimal formation by promoting CXCR4-dependent homing. J Vasc Surg. 2011;53: $1668-78$.

24. Cui B, Huang L, Fang Y, Guo R, Yin Y, Zhao X. Transplantation of endothelial progenitor cells overexpressing endothelial nitric oxide synthase enhances inhibition of neointimal hyperplasia and restores endothelium-dependent vasodilatation. Microvasc Res. 2011;81:143-50.

25. Kyrtatos PG, Lehtolainen P, Junemann-Ramirez M, et al. Magnetic tagging increases delivery of circulating progenitors in vascular injury. JACC Cardiovasc Intervent. 2009;2:794-802.

26. Toma C, Fisher A, Wang J, et al. Vascular endoluminal delivery of mesenchymal stem cells using acoustic radiation force. Tissue Eng Part A. 2011;17:1457-64. 